\title{
Inconformidades nos registros em prontuários: opinião dos trabalhadores de saúde
}

\author{
Nonconformities in medical records: opinion of health workers \\ No conformidades en registros: opinión de los trabajadores sanitarios
}

\section{Resumo}

O objetivo foi verificar a opinião dos trabalhadores de saúde sobre as inconformidades dos registros em prontuários de pacientes hospitalizados. Estudo descritivo-analítico, de abordagem qualitativa, realizado em uma instituição de saúde pública do Brasil. Participaram 30 trabalhadores de saúde de diferentes áreas. Utilizou-se um instrumento autoaplicável, com questões abertas. Os dados foram organizados com o auxílio do software ATLAS ti e a análise de conteúdo de Bardin. Entre as principais inconformidades destacaram-se registros incorretos ou incompletos $(90,9 \%)$, letra ilegível ou rasuras $(27,2 \%)$ e ausência de registros $(12,1 \%)$. Quanto às causas de tais inconformidades identificaram-se, falta de interesse $(54,5 \%)$, desconhecimento da legalidade do registro $(27,2 \%)$, sobrecarga e déficit de recursos humanos $(24,2 \%)$ insuficiência de qualificação e de conhecimento dos trabalhadores sobre a temática $(24,2 \%)$. Diante das evidências apontadas no estudo, recomenda-se rever a política de qualificação dos trabalhadores, aliada à elaboração de protocolos, adequação dos impressos para os registros e organização criteriosa dos prontuários. Palavras-chave: Registros; Prontuário; Inconformidades; Trabalhadores da saúde.

\begin{abstract}
The objective was to verify the opinion of health workers about the non-conformities of records in hospitalized patients' medical records. Descriptive-analytical study, with a qualitative approach, carried out in a public health institution in Brazil. 30 health workers from different areas participated. A self-administered instrument with open questions was used. Data were organized using the ATLAS ti software and Bardin's content analysis. Among the main nonconformities, incorrect or incomplete records (90.9\%), illegible handwriting or erasures $(27.2 \%)$ and absence of records $(12.1 \%)$ were highlighted. As for the causes of such nonconformities, lack of interest (54.5\%), ignorance of the legality of registration (27.2\%), overload and deficit of human resources $(24.2 \%)$ insufficiency of qualification and knowledge were identified. of workers on the subject $(24.2 \%)$. In view of the evidence pointed out in the study, it
\end{abstract}


is recommended to review the workers' qualification policy, together with the elaboration of protocols, adequacy of forms for records and careful organization of medical records.

Keywords: Records; Medical record; Nonconformities; Health workers.

\section{Resumen}

El objetivo fue verificar la opinión de los trabajadores de la salud sobre las no conformidades de los registros en las historias clínicas de los pacientes hospitalizados. Estudio descriptivo-analítico, con abordaje cualitativo, realizado en una institución de salud pública en Brasil. Participaron 30 trabajadores de la salud de diferentes áreas. Se utilizó un instrumento autoadministrado con preguntas abiertas. Los datos se organizaron utilizando el software ATLAS ti y el análisis de contenido de Bardin. Entre las principales no conformidades se destacaron registros incorrectos o incompletos $(90,9 \%)$, caligrafía o tachaduras ilegibles $(27,2 \%)$ y ausencia de registros $(12,1 \%)$. En cuanto a las causas de dichas no conformidades, se identificaron desinterés $(54,5 \%)$, desconocimiento de la legalidad del registro $(27,2 \%)$, sobrecarga y déficit de recursos humanos $(24,2 \%)$ insuficiencia de calificación y conocimientos de los trabajadores en el tema (24,2\%). A la vista de las evidencias señaladas en el estudio, se recomienda revisar la política de calificación de los trabajadores, junto con la elaboración de protocolos, adecuación de los formularios para los registros y una cuidadosa organización de las historias clínicas.

Palabras clave: Registros; Historial médico; No conformidades; Trabajadores de la salud.

\section{Introdução}

O prontuário é um documento destinado aos registros relacionadas aos cuidados prestados aos pacientes durante o seu tratamento de saúde, no âmbito hospitalar ou ambulatorial (Cordeiro, Dyniewicz \& Kallinowski, 2011; Morais, Batista, Castro, Assunção \& Castro, 2015).

A organização do prontuário deve ser feita de forma criteriosa, pois, trata-se de um documento de consulta, avaliação, ensino, pesquisa, ética, comunicação defesa e acusação entre profissionais. Não deve apresentar irregularidades quanto ao seu preenchimento, evitando assim problemas relacionados à comunicação, às pesquisas e à continuidade da assistência (Demarchi et al., 2012).

$\mathrm{O}$ ato de registrar a assistência ao paciente apresenta-se como um meio de comunicação para a equipe de saúde, pois, ao registrar as ações realizadas, a continuidade da assistência torna-se facilitada e ainda auxilia a tomada de decisão (Silva, Grossi, Haddad \& Marcon, 2012).

A comunicação por meio de registros nos prontuários é uma importante ferramenta para uma assistência de qualidade, podendo subsidiar os processos de avaliação da qualidade, monitorar os indicadores de desempenho de serviços, além de legitimar o trabalho do profissional de saúde (Silva, Grossi, Haddad \& Marcon, 2012). Para tanto deve conter informações concisas, completas e fidedignas. Tal situação exige dos profissionais de saúde uma postura inovadora frente aos avanços tecnológicos, à globalização e as mudanças na cultura organizacional em prol de uma práxis segura e de qualidade (Nomura, Silva \& Almeida, 2016).

Os registros devem ser realizados, seguindo as normatizações preconizadas pelos seus respectivos Conselhos Federais da área de atuação profissional, evitando lacunas ou inconformidades, como, o fato de não registrar e ou rasurar os registros nos prontuários que implicam na interrupção do processo assistencial, na dificuldade de comunicação e impossibilita à implementação de ações integralizadas (Barral et al., 2012).

Inconformidades como ilegibilidade, rasuras, ausência de carimbo, checagem da ação, prescrições médicas e de enfermagem, entre outras, comprometem a eficácia dos registros nos prontuários, a qualidade da assistência e a otimização dos custos hospitalares (COFEN, 1986; ambulatorial (Cordeiro, Dyniewicz \& Kallinowski, 2011).

Em relação aos custos hospitalares, a análise dos registros em prontuários permite a verificação da compatibilidade entre o procedimento realizado e os itens que compõem a conta hospitalar (Maia, Barbosa, Silva, Branco, Rodrigues \& Melo, 2017; Padilha, Haddad, Matsuda, 2014). Considerando a importância dos prontuários para a avaliação de custos e qualidade, percebe-se a necessidade da construção de registros livres de inconformidades que representem a assistência prestada (Silva, Grossi, Haddad \& Marcon, 2012). 
O conhecimento equivocado dos profissionais acerca das responsabilidades envolvidas no processo de trabalho deve ser uma preocupação dos gestores da instituição de saúde, quanto aos investimentos na qualificação dos recursos humanos, por meio de uma política de educação continuada com a finalidade de esclarecer a obrigatoriedade dos registros nos prontuários, de forma clara e concisa (Seignemartin, Jesus, Vergílio, Silva, 2013).

Diante das evidências na literatura focada nos registros em prontuários de pacientes hospitalizados e da nossa vivência no cuidado em saúde, deparamo-nos com profissionais alheios ao ato de registrar suas ações. Esse comportamento despertou o nosso interesse em desvelar quais eram as principais inconformidades, bem como, as suas causas relacionadas aos registros nos prontuários, na visão da equipe de saúde.

Nessa perspectiva este estudo teve como objetivo, analisar as inconformidades nos registros em prontuários de pacientes hospitalizados segundo os trabalhadores de saúde.

\section{Metodologia}

Estudo descritivo-analítico, de abordagem qualitativa realizado em um hospital público de grande porte da região central do Brasil, especializado no atendimento materno-infantil (Estrela, 2018).

Participaram do estudo 30 profissionais sendo 20 trabalhadores de saúde da instituição e 10 residentes de enfermagem. Foram incluídos profissionais com vínculo empergatício ou acadêmico da instituição que participaram dos dois módulos de capacitação, sobre os aspectos científicos, éticos e legais da assistência segura e qualidade no contexto assistencial.

O projeto foi aprovado em Comitê de Ética em Pesquisa, sob o protocolo CEPESH/HMI/GO, nº 1.447.390, e assinatura dos sujeitos no Termo de Consentimento Livre e Esclarecido.

A coleta de dados foi realizada em dezembro de 2016. Foi utilizado um instrumento autoaplicável, para conhecer a opinião dos participantes sobre as inconformidades nos registros de prontuários com duas questões:

- Quais são as principais inconformidades encontradas nos registros em prontuários?

- Quais são as principais causas das inconformidades encontradas nos registros em prontuários?

A aplicação do instrumento ocorreu em dois momentos, no início e no final da ação educativa.

A ação educativa foi desenvolvida na própria instituição, no mês de dezembro de 2016, com carga horária total de oito horas. Os participante foram convidados e disponibilizadas 40 vagas, e decorrência da capacidade máxima do espaço físico. Foram oferecidos certificados, material didático, lanche e sorteio de brindes como estratégia de incentivo a participação dos profissionais.

A organização da atividade foi uma atividade coletiva da comissão organizadora do evento, docentes e discentes do programa de pós-graduação Ciências da Saúde da Universidade Federal de Goiás e os gestores do serviço de enfermagem da instituição.

As atividades educativas ocorreram em dois módulos:

No primeiro módulo contextualizou-se os aspectos científicos, éticos e legais no contexto da assistência segura e de qualidade, a importância da qualificação profissional, tomada de decisão, exercício legal da profissão, prestação do cuidado e o registro da assistência de enfermagem no prontuário. Destacou-se o prontuário como fonte de informação para auditoria interna e de avaliação da qualidade da assistência em saúde. A avaliação dos prontuários aconteceu através de um instrumento, validado no Brasil por Haddad (Haddad, 2004)

O segundo módulo foi oferecido cinco dias após o primeiro, em decorrência da disponibilidade do espaço físico destinado a atividade na instituição. Nesse módulo abordou-se, questões referentes à auditoria interna como ferramenta da qualidade da assistência, a responsabilidade do profissional frente aos registros e à otimização dos custos. Apresentou-se ainda 
o PDCA como ferramenta proposta para planejamento e monitoramento de melhorias na qualidade da assistência em serviços de saúde.

Utilizou-se o software ATLAS ti, para a organização dos dados, seguida da sua análise e interpretação, a partir das categorias, representadas por núcleos de sentido e as citações, considerados fragmentos significativos dos relatos dos profissionais. A análise das categorias consolidou a organização das ideias e sua codificação determinada por meio dos núcleos de sentido, conforme Bardin (2011).

\section{Resultados}

A caracterização dos sujeitos ( $n=30)$, mostrou que 24(80\%), eram do sexo feminino e 6(20\%) do sexo masculino. Dos participantes $10(33,4 \%)$ eram residentes de enfermagem e 20(66,6\%) trabalhadores de saúde, sendo 15(75,0\%) enfermeiros, $4(20,0 \%)$ médicos e $1(5,0 \%)$ biomédico.

Com relação às inconformidades foram referidas por $100 \%$ dos profissionais sobre a eficácia dos registros nos prontuários de pacientes hospitalizados. Foram evidenciados por 30\% dos profissionais registros incorretos ou incompletos com letra ilegível e rasuras.

A ausência de identificação do profissional, por meio da assinatura e do carimbo envolvendo o ato de registrar, foi destacada por $13,3 \%$ dos participantes.

Os registros incorretos ou incompletos, letra ilegível e as rasuras na visão dos profissionais foram percebidos como as principais inconformidades em relação aos registros nos prontuários.

Foram destacados por $60 \%$ dos profissionais que tais inconformidades ocorreram pela falta de atenção e de interesse do trabalhador e para $30 \%$ essas ocorrências foram por desconhecimento da legalidade dos registros. Segundo 26,66\% profissionais, a ausência de um programa de capacitação com o objetivo de esclarecimentos sobre o ato de registrar suas ações nos prontuários dos pacientes hospitalizados, contribui para as inconformidades.

Ainda como outros aspectos que comprometem o ato de registrar de forma concisa, completa e correta foram relatados, a sobrecarga de trabalho e ao déficit de recursos humanos; a ausência de fiscalização do prontuário eletrônico e da padronização das ações foram citadas por 3,33\% dos profissionais.

A análise dos dados obtidos antes e após a atividade educativa, envolvendo as inconformidades nos prontuários dos pacientes na visão dos sujeitos da pesquisa, encontra-se demonstradas nas Tabelas 1 e 2 . 
Tabela 1. Principais inconformidades dos registros em prontuários, na percepção dos profissionais (n-30), antes e após ação educativa. Goiânia, 2016.

\begin{tabular}{lclc}
\hline \multicolumn{1}{c}{ INCONFORMIDADES (antes) } & $\mathbf{n}(\%)$ & INCONFORMIDADES (após) & n (\%) \\
\hline Registros incorretos ou incompletos & $30(100 \%)$ & Registros incompletos & $17(56,66 \%)$ \\
& & Letra ilegível e rasuras & $8(26,66 \%)$ \\
Letra ilegível e rasuras & $9(30 \%)$ & & $3(10 \%)$ \\
Ausência de registros e de identificação & $4(13,3 \%)$ & $\begin{array}{l}\text { Ausência de checagem de } \\
\text { procedimento }\end{array}$ & $2(6,66 \%)$ \\
do profissional & & Ausência de registros e de identificação \\
Ausência de clareza & & do profissional & \\
Ausência de checagem de procedimento & $1(3,33 \%)$ & & \\
\hline
\end{tabular}

Fonte: Autores.

Tabela 2. Principais causas de inconformidades nos registros em prontuários, na percepção dos profissionais (n-30), antes e após ação educativa. Goiânia, 2016.

\begin{tabular}{|c|c|c|c|}
\hline $\begin{array}{c}\text { CAUSAS DAS } \\
\text { INCONFORMIDADES } \\
\text { (antes) }\end{array}$ & n (\%) & $\begin{array}{c}\text { CAUSAS DAS } \\
\text { INCONFORMIDADES } \\
\text { (após) }\end{array}$ & $\mathrm{n}(\%)$ \\
\hline $\begin{array}{l}\text { Falta de atenção e de interesse do } \\
\text { trabalhador }\end{array}$ & $18(60 \%)$ & $\begin{array}{l}\text { Ausência de qualificação e de } \\
\text { conhecimento sobre registros } \\
\text { em prontuários }\end{array}$ & $8(26,66 \%)$ \\
\hline $\begin{array}{l}\text { Sobrecarga e déficit de recursos } \\
\text { humanos no serviço }\end{array}$ & $16(53,33 \%)$ & $\begin{array}{l}\text { Sobrecarga de trabalho e } \\
\text { déficit de recursos humanos }\end{array}$ & $7(23,33 \%)$ \\
\hline $\begin{array}{l}\text { Desconhecimento da legalidade do } \\
\text { registro pelo trabalhador }\end{array}$ & $9(30 \%)$ & $\begin{array}{l}\text { Falta de atenção e de } \\
\text { compromisso do trabalhador }\end{array}$ & $4(13,33 \%)$ \\
\hline $\begin{array}{l}\text { Ausência de qualificação e de } \\
\text { conhecimento sobre registros em } \\
\text { prontuários }\end{array}$ & $8(26,66 \%)$ & $\begin{array}{l}\text { Desconhecimento da } \\
\text { legalidade do registro pelo } \\
\text { trabalhador }\end{array}$ & $4(13,33 \%)$ \\
\hline Ausência de fiscalização do superior & $1(3,33 \%)$ & $\begin{array}{l}\text { Ausência de padronização das } \\
\text { ações }\end{array}$ & $1(3,33 \%)$ \\
\hline Ausência de prontuário eletrônico & $1(3,33 \%)$ & $\begin{array}{l}\text { Ausência de comunicação } \\
\text { entre a equipe }\end{array}$ & $1(3,33 \%)$ \\
\hline Ausência de padronização das ações & $1(3,33 \%)$ & & \\
\hline $\begin{array}{l}\text { Ausência de comunicação entre a } \\
\text { equipe }\end{array}$ & $1(3,1 \%)$ & & \\
\hline
\end{tabular}

Fonte: Autores.

\section{Discussão}

A ausência de registros nos prontuários de pacientes hospitalizados ou realização dos mesmos de forma incompleta ou incorreta demonstram inconformidades relacionadas às recomendações legais. Entretanto, estas inconformidades podem ser decorrentes da falta de conhecimento dos profissionais acerca da formalidade dos registros no prontuário do paciente, relativo ao ciclo de cuidados a ele prestados. Registrar as intervenções não pode ser aprendido como um ato voluntário, mas sim como 
um dever ético e legal que serve também para respaldar o profissional diante de processos envolvendo a prática laboral (Padilha, Haddad, Matsuda, 2014).

A Resolução 1638/2002 do Conselho Federal de Medicina define prontuário como "documento único constituído por um conjunto de informações, sinais e imagens registradas, geradas a partir de fatos, acontecimentos e situações sobre a saúde do paciente e a assistência a ele prestada, de caráter legal, sigiloso e científico, que possibilita a comunicação entre os membros da equipe multiprofissional e a continuidade prestada ao indivíduo." Em casos de registros omitidos ou irregulares, o médico pode perder a possibilidade de comprovação de seus atos (CFM, 2002).

No caso dos registros referentes ao cuidado de enfermagem, a Lei 7498, do Conselho Federal de Enfermagem, traz no seu art.14 a incumbência de anotar no prontuário do paciente todas as atividades procedentes da assistência (COFEN, 1986). Dessa forma, os registros de intervenções de enfermagem e suas respectivas ações ou atividades em prontuários fazem parte da responsabilidade legal da enfermagem (COFEN, 1986; Carneiro, Dutra, Costa, Mendes, Arreguy-Sena, 2016; Camelo, Pinheiro, Campos, Oliveira, 20019).

As inconformidades como, letra ilegível e rasuras envolvendo os registros foram destacadas pelos profissionais. Segundo o artigo 299 do Código Penal, a anotação incorreta, incompleta, falseada ou inexistente no prontuário quanto aos fatos relacionados com o paciente pode caracterizar falsidade ideológica. Entre as implicações e abrangência dessas falhas encontram-se as dificuldades relacionadas ao julgamento das ações em processo judicial, pois o juiz poderá emitir seu parecer conforme a fé de seu julgamento (Brasil, 2002).

Do ponto de vista ético e legal, somente o registro da execução de uma atividade pode, de fato, assegurar a sua realização pelo profissional de saúde. Com isso, o registro das atividades é sempre necessário por promover maior visibilidade da categoria profissional e contribuir para a integralidade do cuidado prestado ao paciente (Padilha, Haddad, Matsuda, 2014).

Por outro lado, a falta do carimbo e da assinatura do trabalhador foram outras inconformidades referidas pelos profissionais, que por sua vez, dificultam a identificação do profissional responsável pelo procedimento e inviabiliza a eficácia dos registros nos prontuários do ponto de vista assistencial e jurídico. Isso pode ocorrer pelo fato do profissional, especialmente da equipe de enfermagem, não valorizar as prescrições realizadas pelo enfermeiro. A ausência da identificação do profissional que realizou o procedimento pode gerar dúvidas e resultar em prejuízos à continuidade da assistência e ou dificultar investigações judiciais e éticas futuras, caso sejam necessárias (Padilha, Haddad, Matsuda, 2014).

Nesse contexto, as evidências na literatura recomendam que os registros nos prontuários devam ocorrer de forma clara e concisa, por se tratar de documentos legais que podem comprometer ou defender os trabalhadores e a instituição de saúde em ações judiciais por atos de negligência, imprudência ou imperícia segundo o que determina o Código Civil Brasileiro/2002, no seu Art. 186. Desse modo, entende-se que o registro no prontuário do paciente é fundamental para conferir uma assistência de saúde em conformidade com os princípios legais, de segurança e dos protocolos afins (Seignemartin, Jesus, Vergílio \& Silva, 2013).

Do ponto de vista da gestão do serviço, os profissionais destacaram a sobrecarga de trabalho e déficit de profissionais, bem como, a falta de conhecimento da formalidade do registro de suas ações e falta de qualificação dos profissionais como inconformidades que comprometem os princípios da segurança do paciente. A despeito dessas informações recomenda-se aos gestores estabelecer uma política de educação permanente em conformidade com o preconizado pelo Sistema Único de Saúde (SUS), seguindo a Portaria GM/MS nº 198/2004, que constitui a Política Nacional de Educação Permanente em Saúde (PNEPS) considerada um como um dos seus pilares de sustentação, a formação de seus trabalhadores (BRASIL, 2004; Wensceslau, Albuquerque, 2014).

As inconformidades verificadas nos registros em prontuários, muitas vezes, são justificadas pelo fato dos trabalhadores, não reconhecerem os riscos destas falhas para a assistência do paciente. Igualmente, pelo fato do prontuário ser 
um documento legal, situações que reforçam a necessidade de instituir no serviço, estratégias educativas que possam sensibilizar os profissionais acerca da importância do prontuário e das anotações para a qualidade da assistência. E também, impedir que itens que compõem as contas hospitalares sejam glosados, gerando prejuízos desnecessários ao faturamento da instituição ambulatorial (Cordeiro, Dyniewicz \& Kallinowski, 2011).

O registro em prontuário abrange diversos aspectos e respalda de forma ética e legal o profissional responsável pelo cuidado, assim como, o paciente. Em presença de registros incompletos, letra ilegível e inconsistente, além de não fazer diferencial algum para a equipe multidisciplinar durante o tratamento do paciente, pode influenciar em desperdícios e onerar os custos com a terapêutica para a instituição de saúde.

Por outro lado, o fato do profissional de saúde desconhecer a legalidade dos registros nos prontuários e documentos afins, faz com que este profissional sinta-se desobrigado a registrar suas ações relacionadas ao ciclo de cuidados em conformidade com a legislação, compromete fortemente o processo de trabalho, a segurança do paciente e avaliação da qualidade do serviço (Carneiro, Dutra, Costa, Mendes \& Areguy-Sena, 2016; Morais, Batista, Castro, Assunção \& Castro, 2015).

Nesse sentido, a busca pela qualidade dos registros apresenta-se como um processo complexo que possibilita a identificação de inconformidades e o planejamento de estratégias que possam melhorar a dinâmica de trabalho na instituição (Silva, Loureiro, Frota, Ortega, Ferraz, 2013).

Vale destacar também a baixa assimilação das informações dos profissionais de saúde após a atividade educativa realizada no contexto deste estudo. Este resultado reforça a necessidade da gestão instituir um programa de educação permanente no serviço em conformidade com o Sistema Único de Saúde buscando corrigir e ou minimizar tais inconformidades, considerando a relevância dos registros nos prontuários dos pacientes hospitalizados, no âmbito da gestão da assistência e dos custos para a instituição.

\section{Conclusão}

Os resultados do estudo mostraram inconformidades nos registros de prontuários de pacientes hospitalizados, como inconsistência, rasuras, letra ilegível, falta da assinatura e do carimbo tendo como causas a falta de atenção e de interesse do trabalhador, sobrecarga e déficit de recursos humanos no serviço, desconhecimento da legalidade do registro, ausência de qualificação e de conhecimento sobre registros em prontuários.

Nesse sentido recomenda-se que as instituições de saúde promovam investimentos na capacitação dos recursos humanos a partir do processo de admissão, com instruções e treinamentos direcionados às exigências do processo de trabalho livre de inconformidades. A elaboração e implementação de protocolos de cuidados informatizados, para a prescrição de enfermagem, aliada à adequação dos impressos, contribuem para registros consistentes e de qualidade.

Frente à importância de registros livres de inconformidades, torna-se relevante a realização de novos estudos sobre a essa temática, com o intuito de indicar os principais fatores responsáveis pelas falhas nos registros e subsidiar a elaboração de estratégias consistentes para a melhoria da qualidade da assistência e dos registros em prontuários de pacientes hospitalizados.

\section{Referências}

Bardin, L. (2011). Análise de conteúdo. Edições 70, 229.

Barral, L.N.M, et al. (2012) Análise dos registros de enfermagem em prontuários de pacientes em um hospital de ensino. Rev. Min. Enferm., 16(2): 188-193.

Brasil. (2002) Código Civil, Lei 10.406, de 10 de janeiro de 2002. Revista dos Tribunais.

Brasil. (2004) Ministério da Saúde. Gabinete do Ministro. Portaria nº 198/GM/MS, de 13 de fevereiro de 2004. Institui a Política Nacional de Educação Permanente em Saúde como estratégia do Sistema Único de Saúde para a formação e o desenvolvimento de trabalhadores para o setor e dá outras providências. Brasília: MS. 
Camelo, S. H. H, Pinheiro, A., Campos, D., Oliveira, T. L. (2009). Auditoria de enfermagem e a qualidade da assistência à saúde: uma revisão da literatura. Rev Eletr Enferm, 11(4):1018-25.

Carneiro, S. M., Dutra, H. S., Costa, F. M., Mendes, S. E., Arreguy-Sena, C. (2016). Uso de abreviaturas nos registros de enfermagem em um hospital de ensino. Rev. Rene, ;17(2):208-16.

CFM. Conselho Federal De Medicina. (2002). Define prontuário médico e torna obrigatória a criação da Comissão de Revisão de Prontuários nas instituições de Saúde. Resolução CFM 1.638, de 10 de julho de 2002. CFM.

COFEN. Conselho Federal De Enfermagem. (1986). Dispõe sobre a regulamentação do exercício da Enfermagem e dá outras providências. Lei nº7.498/86, de 25 de junho de 1986.

Cordeiro, F. F., Dyniewicz, A. M., Kalinowski, L.C. (2011). Auditorship in nursing records at na intensive care unit. Rev Enferm UFPE, 5(5):1187-192.

Demarchi, T.M., et al. Auditoria de prontuário do paciente: um processo de aprendizagem e integração institucional. Rev. Administração em Saúde, 2012;14(55):50-56.

Estrela, C. (2018). Metodologia Científica: Ciência, Ensino, Pesquisa. Editora Artes Médicas.

Haddad, M. C. L. (2004). Qualidade da assistência de enfermagem: o processo de avaliação em hospital universitário público. Tese (Doutorado em Enfermagem) - Escola de Enfermagem de Ribeirão Preto da Universidade de São Paulo. Ribeirão Preto, $201 \mathrm{f}$.

Maia, A. B. B., Barbosa, A. B., Silva, M. N. P., Branco, L. M. G. C., Rodrigues, L. M. C., Melo, T. T. C. (2017). Technical And Scientific Compilation About Audit And Quality Management: An Integrative Review. Rev. Enfermagem UFPE., 3(3): 1489-1494.

Morais, C. G. X., Batista, E. M. S., Castro, J. F. L., Assunção, S. S., Castro, G. M. O. (2015). Registros de enfermagem em prontuário e suas implicações na qualidade assistencial segundo os padrões de acreditação hospitalar: um novo olhar da auditoria. Rev. Acred., 5(9):64-84.

Nomura, A. T. G., Silva, M. B., Almeida, M. A. (2016). Qualidade dos registros de enfermagem antes e depois da Acreditação Hospitalar em um hospital universitário. Rev.Latino-Am. Enfermagem, 24: 1-9.

Padilha, E. F., Haddad, M. C. F. L., Matsuda, L. M. (2014). Qualidade dos registros de enfermagem em terapia intensiva: avaliação por meio da auditoria retrospectiva. Cogitare Enferm, 12(2): 239-45.

Seignemartin, B. A., Jesus, L. R., Vergílio, M. S. T. G., Silva, E. M. S. (2013). Avaliação da qualidade das anotações de enfermagem no pronto atendimento de um hospital escola. Rev Rene, 14(6):1123-32.

Silva, J.Á., Grossi, A. C. M., Haddad, M. C. L, Marcon, S. S. (2012). Avaliação da qualidade das anotações de enfermagem em unidade semi-intensiva. Escola Anna Nery, 16(3):576-581.

Silva, R. B., Loureiro, M. D. R., Frota, O. P., Ortega, F. B., Ferraz, C. C. B. (2013). Qualidade da assistência de enfermagem em unidade de terapia intensiva de um hospital escola. Rev Gaúcha Enferm., 34(4):114-120.

Wenceslau, L. D, Albuquerque, P. C. (2014). Educação Permanente em Saúde segundo os profissionais da gestão de Recife, Pernambuco. Trab. Educ. Saúde, 12(2): 425 -441. 